\title{
Restraints in low dimensional organic semiconductor devices at high current densities
}

\author{
Raphael Pfattner ${ }^{\mathrm{a}, \mathrm{b}}$, César Moreno ${ }^{\mathrm{c}}$, Cristobal Voz ${ }^{\mathrm{c}}$, Ramón Alcubilla ${ }^{\mathrm{c}}$, Concepció Rovira ${ }^{\mathrm{a}, \mathrm{b}}$, Joaquim \\ Puigdollers ${ }^{\mathrm{c}, *}$, Marta Mas-Torrent ${ }^{\mathrm{a}, \mathrm{b}, * *}$ \\ ${ }^{a}$ Institut de Ciència de Materials de Barcelona (ICMAB-CSIC), Campus UAB, 08193 Bellaterra, Spain. \\ ${ }^{b}$ Networking Research Center on Bioengineering, Biomaterials and Nanomedicine (CIBER-BBN), 08193 Bellaterra, \\ Spain. \\ ${ }^{c}$ Dept. Enginyeria Electrónica, Universitat Politècnica Catalunya, 08034 Barcelona, Spain \\ and Centre de Recerca en Nanoenginyeria (CrNE), 08028 Barcelona, Spain.
}

\begin{abstract}
The understanding of the charge carrier transport in electronic materials is of crucial interest for the design of efficient devices including specially the restraints that arise from device miniaturization. In this work the performance of organic thin-film and single crystal field-effect transistors with the same active material was studied in detail focusing on the high current density regime, where a pronounced non hysteretic maximum in the transconductance was found. Interestingly, in this operation mode for both, thin films and single crystals, comparable densities of free and gate-induced charge carriers were estimated. Kelvin Probe Microscopy was used to measure the contact potential difference and the electrical field along the transistor channel during device operation exhibiting the formation of local space charges in the high current density regime.
\end{abstract}

Keywords: current saturation at high current densities, organic field-effect transistor, non hysteretic maximum in transconductance, Kelvin Probe Microscopy

\section{Introduction}

Organic field-effect transistors (OFETs) have arisen a great interest over the last years for their industrial potential in low-cost electronics. However, from a fundamental point of view, there are still many aspects related to the transport mechanisms and to the device parameters that need to be further understood since they play a crucial role in the final device performance. Typically, to describe the current-voltage characteristics of an OFET and to extract the field-effect mobility $\left(\mu_{f e}\right)$, the model developed by Horowitz et al. is

\footnotetext{
${ }^{*}$ Corresponding author:

** Principal corresponding author:

Email addresses: joaquim.puigdollers@upc.edu (Joaquim Puigdollers), mmas@icmab.es (Marta

Mas-Torrent)
}

employed [1]. However, in this model it is usually assumed that the $\mu_{f e}$ is constant, when it is known that in real OFETs gate bias $\left(\mathrm{V}_{G}\right)$ dependent mobility is commonly found [2, 3]. Such $\mu_{f e}\left(\mathrm{~V}_{G}\right)$ has been explained by several models mainly based on a distribution of traps [4]. The basic concept in these models is that as the gate voltage increases, more traps are filled and, thus, the mobility increases. Here, we observed such increase of $\mu_{f e}$ with $\mathrm{V}_{G}$ in single crystal and thin-film organic transistors using the same active material, but we additionally found that this mobility reaches a maximum value, after which collapses. This effect is experimentally elucidated in terms of the formation of local space charges on the device at high current densities in the accumulation layer. It should be highlighted that in the emerging trend towards device miniaturization, it is crucial to un- 
derstand the device operation principles at high current densities.

\section{Sample fabrication and characterization}

Dithiophene-tetrathiafulvalene (DT-TTF) [5] was chosen as active material to prepare both thin-film and single-crystal OFETs [6, 7]. OFETs were prepared on highly doped silicon wafers with $200 \mathrm{~nm}$ of thermally grown $\mathrm{Si} / \mathrm{SiO}_{2}$ using both bottom-gate top-contact and bottomgate bottom-contact architectures. For all devices gold was used as source and drain electrodes, which were thermally evaporated through a shadow mask. Fifty nm thick DT-TTF films were thermally evaporated on top of a octadecyltrichlorosilane modified $\mathrm{SiO}_{2}$ surface at a deposition rate of $1-2 \AA /$ s. Single-crystals of DT-TTF were grown from a chlorobenzene solution $(\mathrm{c}=1$ $\mathrm{mg} / \mathrm{ml}$ ) as described previously [7]. Kelvin probe microscopy (KPM) measurements were performed using commercial conductive diamond coated $\mathrm{Si}$ tips (Nanosensors) with a Veeco microscope operated under ambient conditions connecting a Keithley 2636 SourceMeter for OFET characterization [8].

\section{Current voltage characteristics of both thin-film and single crystal OFETs}

The measured transfer characteristics in a linear plot for both, a thin-film DT-TTF OFET (TF1) and a typical single-crystal DT-TTF OFET (SC1) measured sweeping the gate voltage in forward direction are shown in Fig. 1, The channel dimensions for TF1 with linear electrodes were measured to be $\mathrm{W}=1.6 \mathrm{~cm}$ and $\mathrm{L}=200$ $\mu \mathrm{m}$ for channel width and length, respectively. In case of SC1, based on a long plate-like shaped single-crystal of DT-TTF, the channel width and length were found to be $\mathrm{W}=40 \mu \mathrm{m}$ and $\mathrm{L}=96$ $\mu \mathrm{m}$, respectively. These geometrical values lead to typical $\mathrm{W} / \mathrm{L}$ ratios of about 80 and a low value of 0.42 , for thin-film and single-crystal devices, respectively. The field-effect mobility in both the linear and saturation regime was extracted using the methodology proposed by Horowitz et al. [1] and commonly used for OFETs. For TF1 a linear field-effect mobility of about $\mu_{f e, \text { lin }}=0.026$ $\pm 0.001 \mathrm{~cm}^{2} / \mathrm{Vs}$ was calculated (inset Fig. 1(b)) while the field-effect mobility calculated in the saturation regime was found to be $\mu_{f e, s a t}=0.033$ $\pm 0.001 \mathrm{~cm}^{2} / \mathrm{Vs}$ with a threshold voltage of about $\mathrm{V}_{T H}=1.7 \pm 0.2 \mathrm{~V}$ (see supporting material (Supp. Mat.) 1). For SC1, $\mu_{f e, \text { lin }}=1.90 \pm 0.03$ $\mathrm{cm}^{2} / \mathrm{Vs}$ was calculated in the linear regime, while in the saturation regime $\mu_{f e, s a t}$ was found to be $2.04 \pm 0.02 \mathrm{~cm}^{2} / \mathrm{Vs}$ with the threshold voltage of about $\mathrm{V}_{T H}=8.5 \pm 0.1 \mathrm{~V}$ (inset Fig. 1(c) and Supp. Mat.). As expected and in agreement with previous results [7], the field-effect mobility of SC1 is about two orders of magnitude higher compared to TF1, which is related to its higher degree of crystallinity and the absence of grain boundaries. Differences in device performance in all the devices fabricated were not significant.

It is important to notice though that at high gate voltage the extraction of the field-effect mobility for both type of devices exhibit a clear deviation from what is expected from the model. In other words, the source-drain current $\left(\mathrm{I}_{D}\right)$ is not linear any more with the $\mathrm{V}_{G}$ (inset Fig 1 (b) and (c). This effect is much more pronounced in the single-crystal devices. Over the last years such deviation from the linear behavior at high gate voltages with the consequent decrease in mobility has been found, especially in devices with low W/L ratios, such as in long plate-like shaped crystals as in SC1 [11]. Some groups attributed this to contact barriers at the electrodes (i.e. bad matching of the Fermi level of the source electrode with the molecular orbitals or a structural disorder at the interface between the metal and the organic semiconductor) 12 14]. However, this was ruled out in four-terminal measurements, where then it was reported that the bulk mobility is effective at lower gate voltages, while at higher electric fields the transport is restricted at the crystal surface resulting in a lower $\mu_{f e}[15,16]$. Further, this effect was also strongly observed when dielectrics with

\footnotetext{
${ }^{1}$ See supplementary material at http://dx.doi.org/XX for additional information including device preparation and electrical characterization.
} 
high dielectric constant were employed [17]. Then it was suggested that when reaching high current densities, the $\mathrm{I}_{D}$ saturates due to Coulomb interactions [18 20].

\section{Transconductance and field-effect mo- bility}

In order to gain a deeper understanding, the transconductance $\left(\frac{\partial I_{D}}{\partial V_{G}}\right)$ of several devices, both thin-films and single-crystals based on DT-TTF, were analyzed in detail (Supp. Mat.). Fig. 2(a) shows the calculated transconductance at various applied drain voltages ranging from $\mathrm{V}_{D}=0 \mathrm{~V}$ to $\mathrm{V}_{D}=-60 \mathrm{~V}$ in steps of $4 \mathrm{~V}$ for TF1, and Fig. 2(b) shows the transconductance of SC1 calculated with drain voltages ranging from $\mathrm{V}_{D}=0 \mathrm{~V}$ to $\mathrm{V}_{D}=-26 \mathrm{~V}$ in steps of $2 \mathrm{~V}$. The observed characteristics are reproducible, fully reversible and non hysteretic. We can rule out therefore irreversible degradation effects of the OSC. In all cases the transconductance shows an increase at low gate voltages, reaching a maximum and decreasing at high gate bias. In an ideal device, with negligible contact resistance, the transconductance is proportional to $\mu_{f e}$ [2]. Fig. 2(c) and (d) show $\mu_{f e, l i n}\left(\mathrm{~V}_{G}\right)$ calculated in the linear regime for TF1 and SC1, respectively, exhibiting an increase followed by a maximum for both device types, but again much more pronounced for SC1. After this point, increasing the gate bias or/and the drain voltage, but still within the linear regime, results in a decrease of $\mu_{f e, l i n}$ calculated following the same model (indicated by open symbols).

\section{The high current density regime}

The calculated current densities, $\mathrm{j}_{D}$, assuming an accumulation layer thickness of about 5 molecular mono-layers (ML) 2, which for DT-TTF corresponds to an accumulation layer thickness of $\mathrm{d}_{a c c}=7 \mathrm{~nm}$, were found to be almost three orders of magnitude higher in single-crystal than

\footnotetext{
${ }^{2}$ As widely believed, the conduction channel in OFETs in accumulation is formed within the first 2-10 ML near the dielectric interface.
}

in thin-film OFETs, which is in agreement with already published data [21 24]. As shown above, the transconductance maximum was stronger pronounced in SC1 compared to TF1 probably due to this difference in current densities. Since the current density in this work was estimated assuming a channel formed within $5 \mathrm{ML}$ for both thin-film and single-crystal OFETs, maintaining $\mathrm{V}_{G}$ and $\mathrm{V}_{D}$ constant, $\mathrm{j}_{D}$ can be increased by decreasing L. In order to compare the devices thinfilm OFET 2 (TF2) was fabricated with a channel length of $\mathrm{L}=20 \mu \mathrm{m}$ and a channel width of $\mathrm{W}=1 \mathrm{~cm}$, to increase the current density within the accumulation layer. This device exhibited similar values of $\mu_{f e, l i n}$ as in TF1. As anticipated, the calculated transconductance of TF2 showed indeed a stronger pronounced maximum more similar to what was observed in SC1 (see Supp. Mat.). This observation in both device types prompted us to estimate the density of free, gate-induced charges $\left(\mathrm{n}_{\text {free }}\right)$ at the maximum of $\frac{\partial I_{D}}{\partial V_{G}}$. Limiting the extraction to a data set where the devices were working within the linear regime, Fig. 3 shows $\mathrm{n}_{\text {free }}$ estimated for TF2 and SC1. As expected, $\mathrm{n}_{\text {free }}$ increases with both gate and drain bias. Interestingly, for both device types, $\mathrm{n}_{\text {free }}$ at the maximum of $\frac{\partial I_{D}}{\partial V_{G}}$ and at current densities from 11 to $63 \mathrm{~A} / \mathrm{cm}^{2}$ (with $\mathrm{V}_{D}$ from -2 to $-8 \mathrm{~V}$ ) for TF2 and from 20 to 1000 $\mathrm{A} / \mathrm{cm}^{2}$ (with $\mathrm{V}_{D}$ from -1 to $-18 \mathrm{~V}$ ) for $\mathrm{SC} 1$, exhibits values of about $1.0 \times 10^{18} \pm 0.7 \times 10^{18} \mathrm{~cm}^{-3}$ 3. That is, it appears that the maximum mobility value is found at a certain current density for both devices, being thus an intrinsic property of the material. This extraction was performed on a set of thin-film and single crystal devices, exhibiting similar values shown in the Supp. Mat. In this context, the Debye length $\lambda_{D}$ is a fundamental material parameter important to take into account in scaling devices. $\lambda_{D}$ is a measure over which mobile, free charge carriers screen out electric fields and the distance where significant

\footnotetext{
${ }^{3} \mathrm{~A}$ maximum and a minimum value of $\mathrm{n}_{\text {free }}$, assuming a thickness of the accumulation layer of 2.8 and $14 \mathrm{~nm}$ corresponding to $2 \mathrm{ML}$ and $10 \mathrm{ML}$, respectively, would correspond to a variation of a factor about 2.5.
} 
charge separation can occur [25, 26]. It is known that $\lambda_{D}$ is smaller in organic materials compared to inorganic semiconductors most likely due to the low relative permitivity of OSCs [27]. Using $\epsilon_{r}=$ 3 - 4 [27 29], $\lambda_{D}$ at $\mathrm{n}_{\text {free }}=1.0 \times 10^{18} \mathrm{~cm}^{-3}$ reaches a value close to the lattice constants of the OSC $(\sim 2 \mathrm{~nm})$. This observation might be an indication for the localization of charge carriers at high current densities in both device types. This is in agreement with the theoretical model developed by Fratini et al. [17, 19, 20] with rubrene single crystal OFETs with highly polarizable dielectrics, that predicted the presence of Coulomb interactions at high current densities.

Measurements of the Contact Potential Difference (CPD) in the OFET channel by means of Kelvin probe microscopy (KPM) were performed in the operating device. This technique allows to obtain spatially resolved data of the effective potential drop in the channel-electrodes region of an OFET in a non invasive way [8]. Due to the limitations in the maximum scan allowed by the AFM microscope, this analysis could only be performed on TF2 with a channel length of $\mathrm{L}=20 \mu \mathrm{m}$. Fig. 4 depicts the calculated electric field (-dCPD/dx) within the channel of TF2 using the CPD shown in the inset. At $\mathrm{V}_{D}=-2 \mathrm{~V}$ and $\mathrm{V}_{G}-\mathrm{V}_{T H}=$ $12 \mathrm{~V}$, which corresponds to the maximum of $\frac{\partial I_{D}}{\partial V_{G}}$ (Fig, 3), the electric field remains roughly constant indicating a uniform distribution of gate-induced charge carriers in the accumulation layer of the device as expected in the linear regime.

By increasing $\mathrm{V}_{G}-\mathrm{V}_{T H}$ to $-59 \mathrm{~V}$, i.e. increasing the current density within the accumulation layer, but still within the linear regime of device operation according to the OFET model [1], a strong pronounced peak starts to appear at the source electrode. These conditions correspond to high gate bias, with a pronounced decrease in both the transconductance and $\mu_{f e, l i n}$, where the assumption of a linearly distributed charge carrier density along the full transistor channel is violated. By further increasing the current density applying $\mathrm{V}_{D}=-10 \mathrm{~V}$ and $\mathrm{V}_{G}-\mathrm{V}_{T H}$ to $-59 \mathrm{~V}$ the peak of the electrical field close to the source electrode further increases reaching a maximum value of $\mathrm{E}$ $=-2.3 \times 10^{6} \mathrm{~V} / \mathrm{m}$. Thus, under these operation conditions, only a small portion of the electric voltage applied at the drain electrode contributes to the electric field responsible for moving charges along the channel, while most of the electric field is localized in a space charge close to the source electrode. The full electrical characteristics showing the evolution of the electric field for the device with increasing current densities are shown in the Supp. Mat. Taking into account that the characteristics for all voltage sets discussed above are within the linear regime of the widely used OFET model, it can be concluded that at increasing current densities a localized space charge at the source electrode of the OFET is build up, violating the assumption of a uniformly distributed charge carrier density within the transistor channel. This effect was found to have a crucial influence on the overall device behavior and is responsible for the shape of the transconductance and the closely related maximum of $\mu_{f e, l i n}\left(\mathrm{~V}_{G}\right)$ often observed in OFETs. Thus, the widely employed model used to extract $\mu_{f e}$ in OFETs has to be applied with care as pointed out previously [2, 3], specially when reaching the high current density regime.

\section{Conclusion}

To conclude, the calculated transconductance of both thin-film and single crystal OFETs with the same OSC as active material revealed a reproducible non hysterestic maximum in the high carrier density regime exhibiting a localized space charge close to the source electrode measured by KPM. Exceeding this maximum, charge screening effects become more important with further increasing current density possibly due to the reduction of the mean free path of charges and the strong electron-phonon interactions that imply strong phonon scattering of the charge carriers. Considering the trend in device miniaturization space charge effects have to be considered as device performance limiting effects for organic electronics. This work strongly contributes to the understanding of the processes that determine the motion of charge carriers in organic materials, which is of tremendous importance for further im- 
proving the device performance and the development of new potential applications.

\section{Acknowledgments}

The authors thank the ERC StG 2012-306826 e-GAMES, the Networking Research Center on Bioengineering, Biomaterials and Nanomedicine (CIBER-BBN), the DGI (Spain) with project POMAS CTQ2010-19501/BQU, Generalitat de Catalunya 2009SGR516 and TEC 2011-27859C02.

\section{References}

[1] G. Horowitz, R. Hajlaoui, H. Bouchriha, R. Bourguiga, M. Hajlaoui, Adv. Mater. 10 (1998) 923-927.

[2] M. Mottaghi, G. Horowitz, Organic Electronics 7 (2006) 528-536.

[3] C. Reese, Z. Bao, J. Appl. Phys. 105 (2009) 024506-8.

[4] G. Horowitz, M. E. Hajlaoui, R. Hajlaoui, Journal of Applied Physics 87 (2000) 4456-4463.

[5] N. Crivillers, N. S. Oxtoby, M. Mas-Torrent, J. Veciana, C. Rovira, Synthesis 2007 (2007) 1621-1623-.

[6] M. Mas-Torrent, C. Rovira, J. Mater. Chem. 16 (2006) 433-436.

[7] R. Pfattner, M. Mas-Torrent, I. Bilotti, A. Brillante, S. Milita, F. Liscio, F. Biscarini, T. Marszalek, J. Ulanski, A. Nosal, M. Gazicki-Lipman, M. Leufgen, G. Schmidt, L. W. Molenkamp, V. Laukhin, J. Veciana, C. Rovira, Adv. Mater. 22 (2010) 4198-4203.

[8] C. Moreno, R. Pfattner, M. Mas-Torrent, J. Puigdollers, S. T. Bromley, C. Rovira, J. Veciana, R. Alcubilla, J. Mater. Chem. 22 (2012) 345-348.

[9] S. C. B. Mannsfeld, A. Sharei, S. Liu, M. E. Roberts, I. McCulloch, M. Heeney, Z. Bao, Adv. Mater. 20 (2008) 4044-4048.

[10] G. Giri, E. Verploegen, S. C. B. Mannsfeld, S. Atahan-Evrenk, D. H. Kim, S. Y. Lee, H. A. Becerril, A. Aspuru-Guzik, M. F. Toney, Z. Bao, Nature 480 (2011) 504-508.

[11] H. Li, B. C.-K. Tee, G. Giri, J. W. Chung, S. Y. Lee, Z. Bao, Adv. Mater. 24 (2012) 2588-2591.

[12] Y. Takahashi, T. Hasegawa, Y. Abe, Y. Tokura, K. Nishimura, G. Saito, Applied Physics Letters 86 (2005) 063504-3.

[13] Y. Takahashi, T. Hasegawa, Y. Abe, Y. Tokura, G. Saito, Applied Physics Letters 88 (2006) 073504-3.

[14] M. Gruber, E. Zojer, F. Schrrer, K. Zojer, Adv. Funct. Mater. 23 (2013) 2941-2952.

[15] J. Takeya, M. Yamagishi, Y. Tominari, R. Hirahara, Y. Nakazawa, T. Nishikawa, T. Kawase, T. Shimoda, S. Ogawa, Appl. Phys. Lett. 90 (2007) 102120-3.
[16] T. Hasegawa, J. Takeya, Sci. Technol. Adv. Mater. 10 (2009) 024314-.

[17] I. N. Hulea, S. Fratini, H. Xie, C. L. Mulder, N. N. Iossad, G. Rastelli, S. Ciuchi, A. F. Morpurgo, Nature Mater. 5 (2006) 982-986.

[18] C. D. Dimitrakopoulos, S. Purushothaman, J. Kymissis, A. Callegari, J. M. Shaw, Science 283 (1999) 822824.

[19] S. Fratini, H. Xie, I. N. Hulea, S. Ciuchi, A. F. Morpurgo, New Journal of Physics 10 (2008) 033031.

[20] S. Fratini, A. F. Morpurgo, S. Ciuchi, phys. stat. sol. (c) 5 (2008) 718-721.

[21] M. Kiguchi, M. Nakayama, K. Fujiwara, K. Ueno, T. Shimada, K. Saiki, J. J. Appl. Phys. 42 (2003) L1408-L1410.

[22] T. Takenobu, S. Z. Bisri, T. Takahashi, M. Yahiro, C. Adachi, Y. Iwasa, Phys. Rev. Lett. 100 (2008) 066601-.

[23] A. Shehu, S. D. Quiroga, P. D'Angelo, C. Albonetti, F. Borgatti, M. Murgia, A. Scorzoni, P. Stoliar, F. Biscarini, Phys. Rev. Lett. 104 (2010) 246602-.

[24] W. L. Kalb, B. Batlogg, Phys. Rev. B 81 (2010) 035327-.

[25] L. Bergmann, C. Schaefer, Lehrbuch der Experimentalphysik, Festkörper, volume 6, Walter de Gruyter, Berlin-New York, 2005.

[26] G. Horowitz, M. Hajlaoui, Synthetic Metals 122 (2001) 185-189.

[27] M. Raja, B. Eccleston, J. Appl. Phys. 110 (2011) 114524.

[28] H. Bässler, A. Köhler, Unimolecular and Supramolecular Electronics I, volume 312, Springer Berlin / Heidelberg, 2012.

[29] S. Scheinert, G. Paasch, phys. stat. sol. (a) 201 (2004) 1263-1301. 

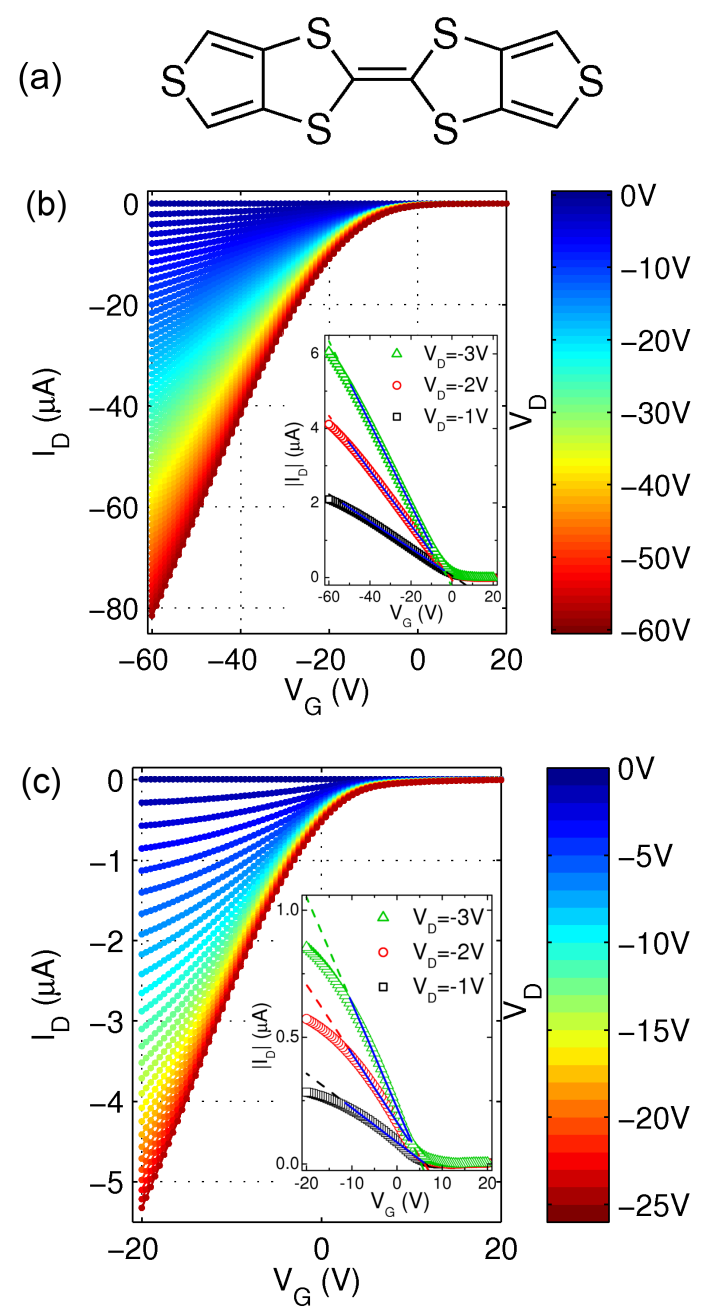

Figure 1: (a) Chemical structure of DT-TTF. Measured transfer characteristics for (b) a typical thin-film based DT-TTF OFET (TF1) (applying $\mathrm{V}_{D}$ in a range from $0 \mathrm{~V}$ to $-60 \mathrm{~V}$ in steps of $1 \mathrm{~V}$ ) with channel width and length, $\mathrm{W}$ $=1.6 \mathrm{~cm}$ and $\mathrm{L}=200 \mu \mathrm{m}$, respectively. (c) Typical DTTTF single-crystal OFET (SC1) (applying $\mathrm{V}_{D}$ in a range from $0 \mathrm{~V}$ to $-26 \mathrm{~V}$ in steps of $1 \mathrm{~V}$ ) with channel width and length, $\mathrm{W}=40 \mu \mathrm{m}$ and $\mathrm{L}=96 \mu \mathrm{m}$, respectively. Insets, extraction of field-effect mobility in the linear regime leading to $\mu_{f e, l i n}=0.026 \pm 0.001 \mathrm{~cm}^{2} / \mathrm{Vs}$ and $\mu_{f e, l i n}=1.90$ $\pm 0.03 \mathrm{~cm}^{2} / \mathrm{Vs}$, for $\mathrm{TF} 1$ and $\mathrm{SC} 1$, respectively.
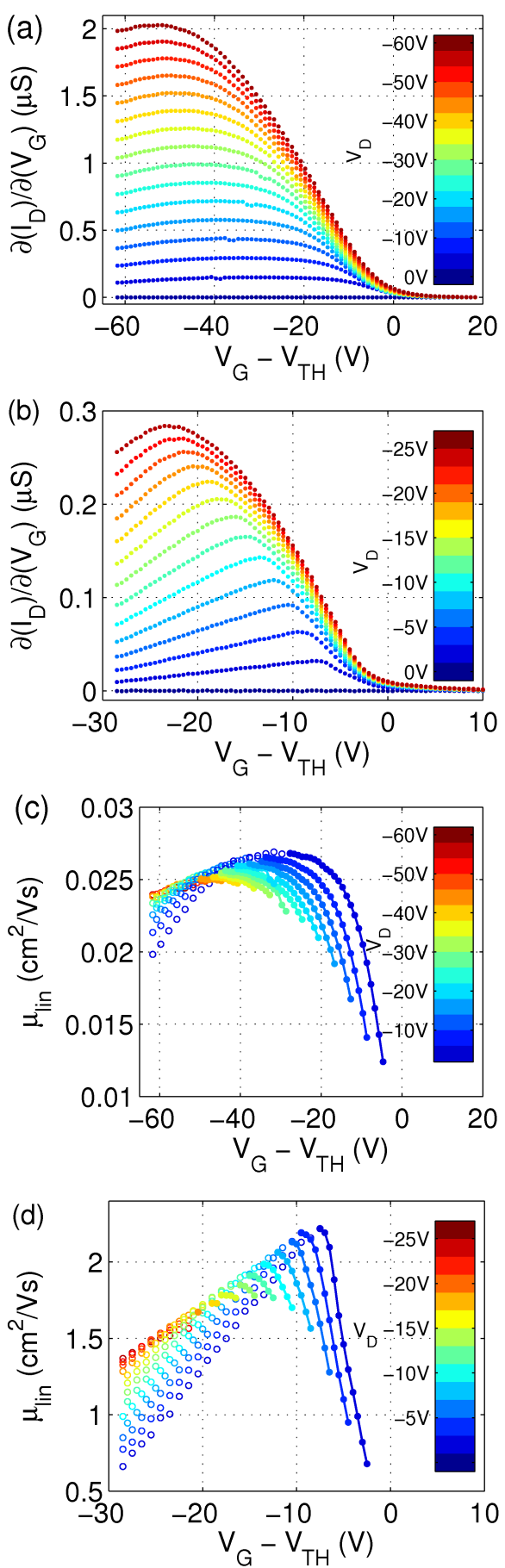

Figure 2: Calculated transconductance $\left(\frac{\partial I_{D}}{\partial V_{G}}\right)$ for (a) TF1, applying $\mathrm{V}_{D}$ in a range from $0 \mathrm{~V}$ to $-60 \mathrm{~V}$ in steps of 4 $\mathrm{V}$ and (b) for $\mathrm{SC} 1$, applying $\mathrm{V}_{D}$ in a range from $0 \mathrm{~V}$ to $-26 \mathrm{~V}$ in steps of $2 \mathrm{~V}$. Gate bias depended mobility in the linear regime, $\mu_{f e, l i n}\left(\mathrm{~V}_{G}\right)$, calculated using the transconductance of (c) TF1 and (d) SC1. Data shown in open symbols correspond to deviation from linear $\mathrm{I}_{D}\left(\mathrm{~V}_{G}\right)$. 


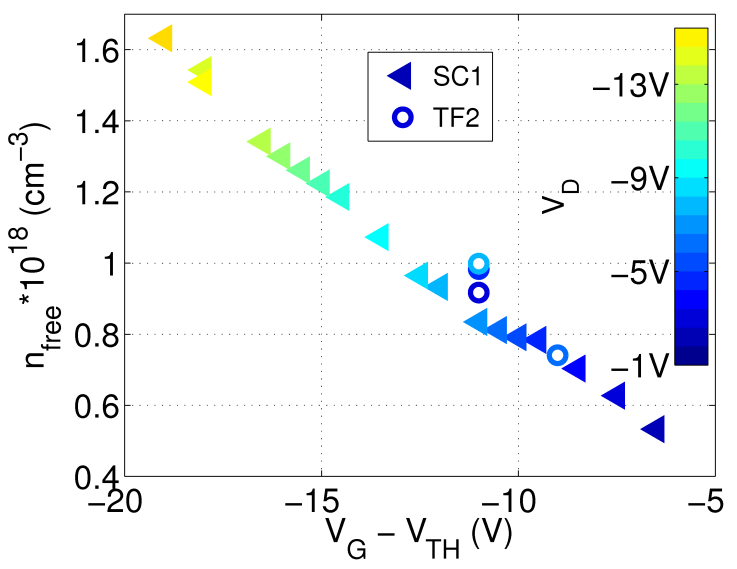

Figure 3: Estimated density of free, gate-induced charge carriers $\mathrm{n}_{\text {free }}$ in the linear regime at the maximum of $\frac{\partial I_{D}}{\partial V_{G}}$ within the accumulation layer calculated for TF2 and SC1 at different $\mathrm{V}_{D}$. Data points extracted for TF2 with $\mathrm{V}_{D}$ $=(-2,-4,-6$ and -8$) \mathrm{V}$.

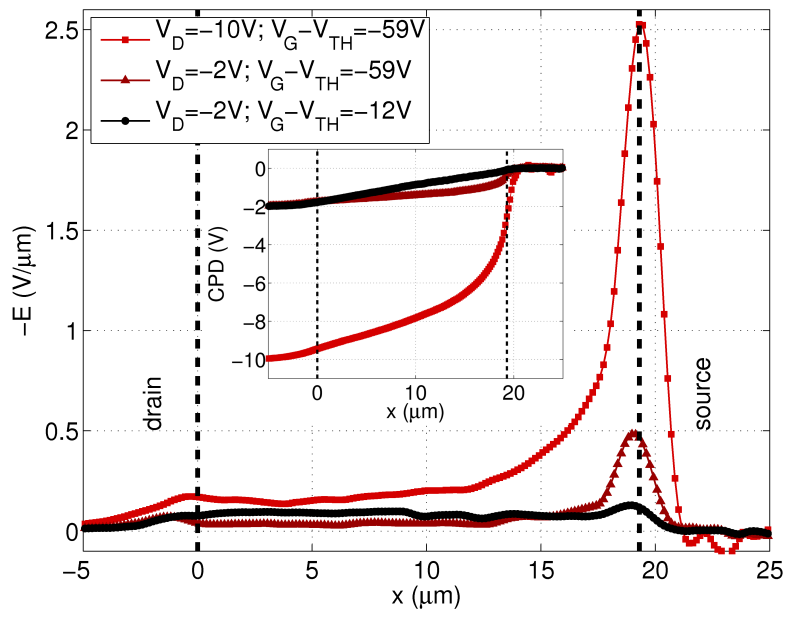

Figure 4: Calculated electric field $\mathrm{E}$ along the channel of TF2 at different $\mathrm{V}_{D}$ and $\mathrm{V}_{G}-\mathrm{V}_{T H}$. The inset shows the Contact Potential Difference (CPD) measured by KPM across the OFET channel. 